\title{
Mathe ist schwierig und Deutsch aktuell: Vergleichende Studie zur Charakterisierung von Schulfächern aus Schülersicht
}

\author{
Ludwig Haag Thomas Götz \\ Universität Bayreuth Universität Konstanzl \\ Pädagogische Hochschule Thurgau
}

\section{Math is Difficult and German up to Date: A Study on the Characterization of Subject Domains from Students'Perspective}

Summary: In the present study different school subject domains are investigated in order to detect differences and similarities between these domains. Concerning the characteristics of domains a questionnaire including 14 categories was adopted. The sample consisted of 1228 students ( $55 \%$ female) in grade levels $8(51 \%)$ and 11 . To investigate the empirical structure of the 7 subject domains under investigation with respect to its characteristics we used correspondence analysis as a dimension-reducing method. Results show that authentic teaching strongly differs across domains and that there are clusters of school subjects showing a similar profile. Further, we found little gender differences with respect to students' characterization of specific domains.

Keywords: School subjects, characteristics of domains

Zusammenfassung: Vorliegende Studie untersucht vergleichend mehrere Schulfächer, um Unterschiede bzw. Gemeinsamkeiten zwischen Fächern bzw. Fachkulturen im Hinblick auf Charakteristika dieser Fächer aufzudecken. Bezüglich der zu untersuchenden Charakteristika von Schulfächern wurde ein Fragebogen mit 14 Kategorien eingesetzt. Die Untersuchung wurde an einer Stichprobe von 1228 Schülern (55\% weiblich) der Jahrgangsstufen 8/11 (51\% der Schüler in Jahrgangsstufe 8) durchgeführt. Um eine multidimensionale Datenstruktur (7 Fächer $\times 14$ Kategorien) anschaulich darzustellen, wurde die Korrespondenzanalyse als dimensionsreduzierende Methode verwendet. Es wird deutlich, dass es einzelnen Fächern unterschiedlich gut gelingt, einen Alltagsbezug herzustellen und dass sich unterschiedliche Fachkulturen finden lassen. Zudem zeigt die Studie, dass sich bezüglich der Charakterisierung von Schulfächern eher geringe Geschlechterunterschiede feststellen lassen.

Schlüsselbegriffe: Schulfächer, Fachkulturen

Im Fachunterricht werden die Schüler in elementare Begriffe und Techniken unserer Kultur eingeführt. Fachunterricht, egal auf welcher Schulstufe, ist ein Abbild des disziplinären Denkens, es geht um fachspezifische Kulturwerkzeuge und um nach Disziplinen gegliederte Kulturgegenstände (vgl. Labudde, 2006). Darüber hinaus soll Unterricht einen über das Fach hinausreichenden Lebensbezug herstel- len. Darauf weisen gerade die Präambeln oder fachübergreifenden Kapitel in den Lehrplänen hin. Exemplarisch sollen hier Aspekte aus dem Lehrplan für das Gymnasium in Bayern (2003) genannt werden: So wird die Bedeutung der Veranschaulichung und Abwechslung hervorgehoben: „Im Unterricht werden die Kernbereiche der jeweiligen Fächer mit ihren unterschiedlichen Erkenntnisansätzen anschaulich 
und fundiert vermittelt. Die Lehrkräfte gestalten, auch im Hinblick auf den Anwendungsbezug, die Lernsituationen methodisch vielfältig und der Komplexität der Lerngegenstände angemessen" (S. 9). Anstrengungsbereitschaft und Meinungsaustausch werden für alle Fächer gefordert: „Unerlässlich für die Schüler des Gymnasiums ist der Erwerb überfachlicher Kompetenzen. $\mathrm{Zu}$ diesen zählen vor allem Selbstkompetenz (z. B. Leistungsbereitschaft, Ausdauer ...), Sozialkompetenz (z. B. Kommunikationsfähigkeit, Teamfähigkeit). ... Die Einübung und langfristige Aneignung dieser Kompetenzen tragen sowohl zur Verbesserung der Arbeitsqualität als auch wesentlich zur Formung einer gefestigten Persönlichkeit bei“ (S. 10).

Auch konkret für einzelne Fächer werden solche Lernziele formuliert, wie hier exemplarisch aus dem Lehrplan für Mathematik das Ziel Meinungsaustausch und Lebensbezug angeführt wird: „Ihnen [den Schülern] soll bewusst werden, dass viele Probleme unserer Zeit einen rationalen Zugang besitzen, dass mathematische Denk- und Vorgehensweisen Anwendung in den meisten Wissenschaften, den unterschiedlichsten Berufsfeldern und nicht zuletzt in unserem Alltag finden. Ziel ist es, Mathematik als eine lebendige Wissenschaft möglichst vielen jungen Menschen verständlich zu machen, ... Gespräche darüber anzuregen sowie ihre Neugier zu erhalten“ (S. 46). „Dem Praxisbezug und dem Lernen in sinnstiftenden Kontexten muss genügend Zeit eingeräumt werden" (S. 47).

Die exemplarisch aus einem Lehrplan eines Bundeslandes angeführten Lernziele zeigen, dass ein Lehrer mehr sein muss als ein Experte für den Inhalt seines Faches. Und genau dies betonen die vorliegenden Erkenntnisse zum Expertenansatz. In Anlehnung an Shulman (1987) stellt Bromme (1992) eine Topologie professionellen Wissens bei Lehrern zusammen. Er unterscheidet Inhaltswissen, curriculares Wissen, Philosophie des Schulfachs, pädagogisches Wissen und fachspezifisch-pädagogisches Wissen.
So sind in vorliegendem Kontext gerade die Philosophie des Schulfachs und das pädagogische Wissen relevant (vgl. Bromme \& Haag, 2004). Bei der Philosophie des Schulfachs geht es um die Überzeugungen über den Sinn und Zweck eines Schulfachs im schulischen und außerschulischen Zusammenhang. Im Mathematikunterricht beispielsweise lernen die Schüler, ob ihr Lehrer der Überzeugung ist, dass der Kern der Mathematik aus Operationen mit einer klar vorab definierten Sprache besteht oder aus wechselseitigen Beziehungen verwendeter Zeichen oder dass Mathematik vorrangig ein Werkzeug zur Beschreibung der Wirklichkeit ist. In den Naturwissenschaften und in Mathematik konnte anhand zahlreicher Studien nachgewiesen werden, dass solche generellen Überzeugungen die Unterrichtspraxis beeinflussen (z. B. die Art der Lehrererklärungen oder die Integration der Schüleräußerungen in den aktuellen Stoff, vgl. Brickhouse, 1990; Köller, Baumert \& Neubrand, 2000).

Unter pädagogischem Wissen ist fächerübergreifendes Wissen über die Gestaltung des Unterrichtsablaufs, die gemeinsame Stoffentwicklung, die Strukturierung von Unterrichtszeit und über das Klassenmanagement (Aufrechterhaltung von Disziplin) zu verstehen ebenso wie Wissen über allgemeine Lehrmethoden, den Einsatz von Medien und Sozialformen des Unterrichts. Zu untersuchen inwieweit Fachkulturen vorliegen, ist wohl eine Forderung des Expertenansatzes, doch nicht im Detail untersucht (vgl. Bromme \& Haag, 2004). Bei der Gestaltung des konkreten Unterrichts geht es bei jedem Fach um die Balance zwischen einer Strukturorientierung und Anwendungsorientierung. Dabei fallen die Anteile je nach Fach unterschiedlich aus: Gellert (2006) bewertet den heutigen Mathematikunterricht so: „Zwar dienen Praxisbezüge als Anknüpfungspunkte für mathematische Begriffsbildung, doch fungieren die außermathematischen Situationen hierbei lediglich als Motivationsversuche" (S. 468). Reinhold (2006, S. 481) konstatiert für den Physikunterricht ähnlich, dass der gegenwärtige Unterricht die Physik 
überwiegend als Methode darstellt, dass die Physik in ihrer Bedeutung für die Gesellschaft im Unterricht zu wenig thematisiert wird.

Außerdem sollen die Fächer stets der Persönlichkeitsentwicklung dienen, wie der Stärkung von Selbstbewusstsein, Sozialkompetenz und Teamfähigkeit. Und der Anteil offener, eher schülerzentrierter Unterrichtsformate, deren Potenzial gerade in der Ausbildung von sozialen Fertigkeiten gesehen wird (vgl. Slavin, 1995), wird über die Fächer hinweg immer noch angemahnt. Stellvertretend steht die Analyse über das Fach Chemie: Offenere Formen oder Ansätze, die eine bewusste Methodenvielfalt zugrunde legen, sind eher noch selten (Demuth, 2006).

Eine Studie über den zeitlichen Anteil der Unterrichtsmethoden in den Fächern $(N=720$ Schüler; Götz, Lohrmann, Ganser \& Haag, 2005) zeigt, dass hier Fächer eigene Schwerpunkte haben. So dominiert bei den naturwissenschaftlichen und sozialwissenschaftlichen Fächern im Vergleich zu den Sprachen und Mathematik die Gruppenarbeit, wohingegen Einzelarbeit weniger eingesetzt wird.

Diese Studie zeigt, dass es offensichtlich ist, dass es gewisse Unterschiede, aber auch Zusammenhänge zwischen verschiedenen Schulfächern gibt. Allerdings stellt sich die Frage, wo genau diese Verschiedenheiten bzw. Gemeinsamkeiten liegen und was die verschiedenen Schulfächer im Grunde charakterisiert. Auf die Wichtigkeit solcher Untersuchungen, insbesondere für die Didaktik, weist auch Tenorth (1999, S. 195-196) hin. Er bedauert, dass die Fächer „[...] in ihrer heterogenen Binnenstruktur bisher wenig erforscht [sind], weder systematisch, noch empirisch und historisch" (Tenorth, 1999, S. 196). Zudem werden in den wenigen Untersuchungen bezüglich bestimmter Eigenschaften von Domänen die Schulfächer meist getrennt voneinander erforscht (Stevens et al., 2005, S. 127). Beispielsweise werden Merkmale bzw. Eigenschaften eines Schulfaches häufig in der zugehörigen Fachdidaktik dargestellt, üblicherweise völlig separiert von anderen Schulfächern, weshalb Vergleiche kaum möglich sind.
Es lässt sich konstatieren, dass nur vereinzelt Studien existieren, die sich direkt oder indirekt mit allgemeinen Eigenschaften von Schulfächern befassen bzw. Anregungen zu geeigneten Charakteristika geben. Beispielsweise untersuchte Bachmair (1969) die Einstellung von Schülern zum Lehrer und zu den Unterrichtsfächern Mathematik, Deutsch, Englisch, Erdkunde, Musik und Turnen. Mittels eines Fragebogens wurden bei den Schülern zum Beispiel die Beliebtheit des Faches, das Interesse am Fach, die Wichtigkeit des Faches, der Arbeitsaufwand für das Fach, die Kompetenz in diesem Fach und auch das Angsterleben in diesem Fach erhoben. Allerdings wird nicht genauer erläutert, warum gerade diese Kategorien verwendet wurden. Zudem standen in dieser Studie weniger die Fächereigenschaften und Fächerähnlichkeiten im Fokus, sondern vielmehr der $\mathrm{Zu}$ sammenhang der Schülereinstellung zum Fach mit dem Lehrerbild.

Relativ viele Ergebnisse liegen vor über die Beliebtheit von Unterrichtsfächern. In einer repräsentativ angelegten Erhebung der ShellJugendstudie aus dem Jahre 1992 (Shell-Jugendwerk, 1992), in der nach den Lieblingsfächern gefragt wurde, wird deutlich, dass die 13- bis 16-Jährigen die Sprachen Englisch und Deutsch sowie Biologie und Mathematik eher vorne platzieren, Physik und Chemie eher abgeschlagen am Ende auftauchen.

In einer aktuellen Studie von Jäger-Flor und Jäger (2008) wurden die Gesichtspunkte „Beliebtheit“ und „Bedeutung für das spätere Leben" für eine Vielzahl von Fächern erhoben. Dabei schnitten beispielsweise die Kernfächer Englisch und Deutsch in beiden Kategorien sehr gut ab, und auch Mathematik lag relativ weit vorne, im Gegensatz zu den Nebenfächern Kunst, Religion oder Musik.

In sämtlichen Untersuchungen zur Fächerbeliebtheit ist der Einfluss des Geschlechts unübersehbar. Schon Stern (1905) berichtet seine Ergebnisse getrennt für Jungen und Mädchen. Die große Querschnittsuntersuchung von Hoffman und Lehrke (1985) zeigt, dass die Jungen einen Interessenvorsprung in Physik, 
Technik, Chemie und Mathematik haben, Mädchen in Fremdsprachen, Kunst, Deutsch und Biologie. Roisch (2003) betrachtete verschiedene Interessentests und Studien zum Sach- und Fachinteresse (PISA 2000: Stanat \& Kunter, 2001; PISA 2003: Zimmer, Burba \& Rost, 2004; TIMS-Studien: Baumert \& Lehmann, 1997; Baumert, Bos \& Watermann, 1999) in Bezug auf Geschlechterunterschiede und stellt zusammenfassend fest, „[...] dass sich der Tendenz nach Mädchen noch immer eher für den sprachlich-fremdsprachlichen Bereich und Jungen für den mathematisch-naturwissenschaftlichen Bereich interessieren" (Roisch, 2003, S. 149). Allerdings wird diese Aussage durch eine differenzierte Betrachtung der einzelnen Fächer etwas relativiert. So scheint die oben genannte Zweiteilung in besonderem Maße für Deutsch und Physik Bestand zu haben. Denn Englisch finden auch Jungen sehr interessant und genauso können Biologie und Mathematik in Bezug auf die Interessen nicht eindeutig Mädchen oder Jungen zugeordnet werden (Roisch, 2003, S. 150).

Seit dem eher mäßigen Abschneiden in der TIMS-Studie wurden schwerpunktartig im deutschsprachigen Raum Mathematik und die naturwissenschaftlichen Fächer untersucht, sodass über diese Fächer die detailliertesten Erkenntnisse vorliegen. So wertete Merzyn (2008) das mittlerweile umfangreiche Material aus. Die vorliegenden Erkenntnisse wie aus obiger Shellstudie werden bestätigt. Chemie und Physik liegen weit hinten in der Beliebtheitsskala. In Mathematik streuen die Ergebnisse mehr, bei einem Teil der Schüler ist sie sehr beliebt, bei einem anderen sehr unbeliebt. Auch nimmt - mit Ausnahme in Biologie - das Interesse über das Alter hin ab. Auch international längsschnittlich angelegte Studien bestätigen diesen Trend. Psychologen wie Todt (1978) deuten diese Muster weniger als eine schleichende Schulverdrossenheit als vielmehr einen natürlichen Entwicklungsvorgang. Merzyn versucht im Folgenden Gründe für die geringe Akzeptanz gerade von Chemie und Physik aufzuzeigen.
- Belegt sind das Fehlen von Anwendungen und der Abstand des Unterrichts zur Lebenswelt. Dies konnten Krause und Reiners-Logothetidou (1979) feststellen, als sie die physikalischen Kenntnisse von 2718 Studienanfängern der Physik bundesweit erfassten. Hierin liegt nach Merzyn ein Grund für die mangelnde Beliebtheit. Während sich die Schüler nur wenig für die „Kerninhalte“ der Fächer Physik und Chemie begeistern können, finden sie eher den Alltagsbezug und die Anwendung interessant. „Auswahl und Anordnung der Themen folgen im Grundcharakter dem Vorbild von Hochschulvorlesungen und -lehrbüchern" (Merzyn, 2008, S. 51).

- Auffallend groß ist die Kluft zwischen dem geäußerten Interesse und den Einstellungen zur Bedeutung von Chemie und Physik. Offensichtlich gelingt es im naturwissenschaftlichen Unterricht nicht, bei der großen Bedeutung, die den Fächern an sich zukommt, ein Interesse für die Fächer zu wecken. Muckenfuß (1995) spricht von einer „gespaltenen Haltung“, von einem ,zerrissenen Urteil“ (S. 84).

- Methodisch sind die Fächer eingeengt. Es dominiert eine relativ starke Lehrerzentriertheit und eine Unterrichtsführung, die zu kleinschrittig eng ist (Alt-Stutterheim, 1980; Labudde \& Pfluger, 1999; Seidel \& Prenzel, 2004). „Das Lernen ist wenig selbstbestimmt; das Schüler-Bedürfnis nach Autonomie wird nicht befriedigt" (Merzyn, 2008, S. 66).

- Nach Schüleransicht ist die Schwierigkeit in Chemie und Physik sowie Mathematik im Vergleich zu anderen Fächern ungewöhnlich hoch. Und dieser Abstand wächst noch mit den Schuljahren (Euler, 1982).

- Lernerfolge im Physik- und Chemieunterricht sind gering, wie Lehrerurteile, Schülerselbsteinschätzungen sowie Leistungstests belegen (vgl. TIMSS: Baumert \& Lehmann, 1997). 
Allein auf das Fach Mathematik bezogen wurden in der bereits erwähnten Studie von JägerFlor und Jäger (2008) den Befragten zur Einschätzung des Faches Mathematik acht Gegensatzpaare von Begriffen vorgegeben: anschaulich - abstrakt, praxisbezogen - theoriebezogen, leicht zu verstehen - schwer zu verstehen, für die Allgemeinheit - nur für Fachwissenschaftler, interessant - uninteressant, eine spannende Herausforderung - eine lästige Pflicht, absolut logisch - völlig unlogisch und wichtig - unwichtig. Die untersuchten Aspekte sowie die Ergebnisse deuten an, dass sich Mathematik zum Beispiel durch eine hohe Abstraktheit und einen starken Theoriebezug auszeichnet. Leider fehlt der Vergleich zu anderen Fächern. Zudem geben Jäger-Flor und Jäger keine fundierte Begründung für die Wahl der untersuchten Aspekte.

Zusammenfassend lässt sich konstatieren, dass in den Lehrplänen klare Vorstellungen über den "Geist" von Fächern formuliert werden, dass im Expertenansatz eine Topologie professionellen Wissens herausgearbeitet wurde, die über das Fachwissen hinausreicht, und dass vereinzelt Studien vorliegen, die sich mit Eigenschaften von Fächern auseinandersetzen. Diese sind aber häufig unzureichend und lassen einen Ähnlichkeitsvergleich der Fächer nicht zu. Seit PISA 2000 liegt ein ausführlicher Vergleich der beiden Fächer Mathematik und Deutsch vor, worauf die von uns gewählte Überschrift abzielt: „Im Fach Mathematik erleben sich die Schülerinnen und Schüler aller Schulformen wesentlich häufiger als überfordert als im Fach Deutsch" (Deutsches PISA-Konsortium, 2001, S. 500). Wie hier werden häufig nur sehr wenige oder ganz bestimmte Domänen betrachtet. Zudem mangelt es an fundierten Begründungen für die untersuchten Kategorien oder Aspekte, wodurch diese oft sehr willkürlich erscheinen. Deshalb gingen der vorliegenden Studie eine explorative Interviewstudie und eine Fragebogenstudie voraus. Mittels dieser empirischen Verfahren wurden dann geeignete Kategorien zur Charakterisierung von Schulfächern herausgearbeitet (siehe dazu Schnell, 2009).

\section{Fragestellung}

Aufgrund der gleichzeitigen Berücksichtigung verschiedener Schulfächer interessieren folgende Fragestellungen:

1. Inwieweit gelingt es den einzelnen Fächern, einen Lebens-/Alltagsbezug herzustellen, wie vom „Geist des Lehrplans“ gefordert?

2. Inwieweit lassen sich unterschiedliche Fachkulturen finden, in denen sich einzelne Fächer näher sind und von anderen unterscheiden?

3. Inwieweit lassen sich zwischen den Fächern geschlechtsspezifische Unterschiede feststellen?

Diese Fragen werden in vorliegender Studie „nur“ aus Schülerperspektive beantwortet, was den Aussagewert zwangsläufig einschränkt. Wohl wissend, dass Schülerantworten, wenn es um Erreichen von Bildungszielen geht, allein zu kurz greifen, so stellen sie dennoch eine zentrale Informationsquelle dar.

\section{Methodik}

\section{Stichprobe, Datenerhebung und Erhebungsinstrumente}

Es wurden Daten an 1228 Schülern (55\% weiblich) aus 52 Klassen (25/27 Klassen in Jahrgangsstufe 8/11; $50.6 \%$ der Schüler in Jahrgangsstufe 8) an 9 Gymnasien in Baden-Württemberg erhoben. Das Durchschnittsalter der Befragten lag bei 15.88 Jahren $(S D=1.60)$. Die Datenerhebung fand von Anfang Mai bis Mitte Juni 2009 durch geschulte Testleiter während der regulären Unterrichtszeit statt und dauerte pro Klasse ca. 25 Minuten. Voraussetzung für die freiwillige Teilnahme an der Studie war die schriftliche Einwilligung der Eltern bzw. Erziehungsberechtigten. Den Schülern wurde versichert, dass die Daten vollkommen anonymisiert ausgewertet werden und den Lehrkräften keine Einsichtnahme in die Fragebögen möglich ist.

Es wurde die folgende Gruppe von 7 Unterrichtsfächern untersucht, die eine große Bandbreite an Kompetenz- und Inhaltsbereichen abdeckt und sowohl Haupt- als auch Nebenfächer beinhaltet: Mathematik, Physik, Biologie, Deutsch, Englisch, Ge- 
schichte und Musik. Bezüglich der zu untersuchenden Charakteristika von Schulfächern wurden die von Schnell (2009) auf der Basis explorativer Interviews an 40 Gymnasiasten (50\% weiblich, 8. und 11. Jahrgangsstufe) generierten Kategorien verwendet. Bei dieser Studie wurden den Schülern im Interview-Hauptteil 7 Fragen gestellt: „Welche Eigenschaften kennzeichnen für Dich speziell das Fach [Domäne]“ - mit Bezug auf die 7 oben genannten Domänen. Aus den qualitativen Interviewaussagen wurden anhand eines von Mayring und Gläser-Zikuda (2005) vorgeschlagenen Verfahrens distinkte Kategorien gebildet. In Induktions-Deduktionsschleifen wurden die Aussagen dem Kategoriensys- tem zugeordnet, und das Kategoriensystem wurde optimiert und erweitert. Am Ende dieses Prozesses fanden sich 14 unterschiedliche Kategorien. Dass die Schüleraussagen dem Kategoriensystem von unterschiedlichen Personen eindeutig zugeordnet werden können, verdeutlicht die sehr hohe Interraterreliabilität, die in dieser Studie berichtet wird (mittleres Kohen's Kappa über alle Kategorien: .85, 2 Rater) Es handelt sich somit um trennscharfe und aus der Perspektive der Schüler, die diese Kategorien genannt haben, saliente Charakteristika von Schulfächern.

Die 14 Kategorien bildeten die Grundlage zur Entwicklung des in der vorliegenden Studie einge-

Tabelle 1: Erhobene Charakteristika der Fächer

\begin{tabular}{|c|c|c|}
\hline Kategorie & Frage & Skala \\
\hline 1. Stoffmenge & $\begin{array}{l}\text { Ist die Menge des Stoffes, die Du lernen musst, eher } \\
\text { wenig, genau richtig oder viel? }\end{array}$ & $\begin{array}{l}1 \text { wenig } \\
5 \text { viel }\end{array}$ \\
\hline $\begin{array}{l}\text { 2. Hilfsmittel- } \\
\text { benutzung }\end{array}$ & $\begin{array}{l}\text { Werden im Unterricht Hilfsmittel wie zum Beispiel } \\
\text { Taschenrechner, Musikinstrumente, Landkarten oder } \\
\text { Mikroskope verwendet? }\end{array}$ & $\begin{array}{l}1 \text { wenig } \\
5 \text { viel }\end{array}$ \\
\hline $\begin{array}{l}\text { 3. Wichtigkeit } \\
\text { der Note }\end{array}$ & $\begin{array}{l}\text { Wie wichtig ist es Dir, in diesem Fach eine gute Note } \\
\text { zu bekommen? }\end{array}$ & $\begin{array}{l}1 \text { nicht wichtig } \\
5 \text { wichtig }\end{array}$ \\
\hline $\begin{array}{l}\text { 4. Wichtigkeit } \\
\text { des Faches }\end{array}$ & Wie wichtig ist Dir das Fach unabhängig von der Note? & $\begin{array}{l}1 \text { nicht wichtig } \\
5 \text { wichtig }\end{array}$ \\
\hline 5. Anstrengung & $\begin{array}{l}\text { Wie anstrengend empfindest Du den Unterricht in die- } \\
\text { sem Fach? }\end{array}$ & $\begin{array}{l}1 \text { wenig } \\
5 \text { viel }\end{array}$ \\
\hline 6. Alltagsbezug & $\begin{array}{l}\text { Haben die Unterrichtsthemen wenig oder viel Bezug } \\
\text { zum Alltag? }\end{array}$ & $\begin{array}{l}1 \text { wenig } \\
5 \text { viel }\end{array}$ \\
\hline $\begin{array}{l}\text { 7. Zusammenhang } \\
\text { der Themen }\end{array}$ & $\begin{array}{l}\text { Werden im Unterricht eher unzusammenhängende } \\
\text { Themen behandelt oder besteht ein Zusammenhang } \\
\text { der Themen? }\end{array}$ & $\begin{array}{l}1 \text { unzusammenhängend } \\
5 \text { zusammenhängend }\end{array}$ \\
\hline 8. Schwierigkeit & $\begin{array}{l}\text { Empfindest Du die Schwierigkeit in diesem Fach als } \\
\text { eher gering oder als eher hoch? }\end{array}$ & $\begin{array}{l}1 \text { gering } \\
5 \text { hoch }\end{array}$ \\
\hline $\begin{array}{l}\text { 9. Meinungs- } \\
\text { austausch }\end{array}$ & $\begin{array}{l}\text { Gibt es wenig oder viel Meinungsaustausch in diesem } \\
\text { Fach? }\end{array}$ & $\begin{array}{l}1 \text { wenig } \\
5 \text { viel }\end{array}$ \\
\hline $\begin{array}{l}\text { 10. Begabung } \\
\text { erforderlich }\end{array}$ & $\begin{array}{l}\text { Kann man mit Fleiß gute Ergebnisse in diesem Fach } \\
\text { erzielen oder ist eher Begabung notwendig? }\end{array}$ & $\begin{array}{ll}1 & \text { Fleiß } \\
5 & \text { Begabung }\end{array}$ \\
\hline $\begin{array}{l}\text { 11. Veranschauli- } \\
\text { chung }\end{array}$ & Wird in diesem Fach wenig oder viel veranschaulicht? & $\begin{array}{l}1 \text { wenig } \\
5 \text { viel }\end{array}$ \\
\hline 12. Richtige Lösung & $\begin{array}{l}\text { Sind die richtigen Lösungen in diesem Fach uneindeu- } \\
\text { tig oder eindeutig? }\end{array}$ & $\begin{array}{l}1 \text { uneindeutig } \\
5 \text { eindeutig }\end{array}$ \\
\hline 13. Aktuelle Themen & $\begin{array}{l}\text { Werden in diesem Fach wenige oder viele aktuelle The- } \\
\text { men behandelt? }\end{array}$ & $\begin{array}{l}1 \text { wenig } \\
5 \text { viel }\end{array}$ \\
\hline 14. Abwechslung & $\begin{array}{l}\text { Empfindest Du den Unterricht als wenig abwechs- } \\
\text { lungsreich oder als abwechslungsreich? }\end{array}$ & $\begin{array}{l}1 \text { wenig } \\
5 \text { viel }\end{array}$ \\
\hline
\end{tabular}


setzten quantitativen Fragebogens. Alle in der Studie von Schnell (2009) gefundenen Kategorien und die aus ihnen entwickelten Fragebogen-Items sind in Tabelle 1 dargestellt. Sie wurden in der in Tabelle 1 aufgeführten Reihenfolge anhand eines vollstandardisierten Fragebogens (Schülerselbstbericht) in den genannten 7 Unterrichtsfächern erfasst. Dementsprechend lautete die erste Frage im Fragebogen: „Ist die Menge des Stoffes, die Du lernen musst, eher wenig, genau richtig oder viel?" Die Schüler konnten dann für die 7 Fächer auf einer Skala von 1 bis 5 (wenig - viel; die Werte 2, 3 und 4 waren nicht benannt) die entsprechenden Werte angeben. Die Reihenfolge der Fächer war bezüglich aller Fragen die Folgende: (1) Mathematik, (2) Musik, (3) Englisch, (4) Physik, (5) Geschichte, (6) Deutsch, (7) Biologie. Für die Interpretation der Werte ist es wichtig zu betonen, dass die Skalierungen für alle Items von 1 bis 5 gingen, diese sich jedoch im Wortlaut zum Teil unterschieden (z. B. Stoffmenge: wenig - viel; Schwierigkeit: gering hoch).

\section{Datenstruktur und Analysemethoden}

Da bei der vorliegenden Studie Schüler in Klassen untersucht wurden, handelt es sich um eine genestete Datenstruktur (Zwei-Ebenenstruktur). Die Nestung der Klassen in Schulen wurde aufgrund der geringen Zahl unterschiedlicher Schulen $(N=9)$ nicht berücksichtigt. Zudem handelt es sich nicht um unterschiedliche Schultypen (ausschließlich Gymnasien). Um das Ausmaß der Klumpung der Stichprobe einschätzen zu können, wurden 98 Intraklassenkorrelationen (14 Kategorien $\times 7$ Fächer) mit der Software Mplus 5.2 (Muthén \& Muthén, 1998 - 2008) berechnet (Berechnung jeweils anhand des Nullmodells). Die ICCs liegen zwischen .01 und .27 mit einem Mittelwert von .11 und einem Median von .10. Die mittleren Intraklassenkorrelationen für die 14 Kategorien (jeweils Mittelwerte über die 7 Fächer) liegen zwischen .05 und .16, wobei die Intraklassenkorrelationen für eher stark „subjektive“ Kategorien in der Regel niedriger sind als für „offensichtlichere" Kategorien (z. B. .05 bei „Wichtigkeit der Note“, .16 für „Stoffmenge“). Die Mehrebenenstruktur der Daten wird bei der vorliegenden Studie bei jenen Analysen berücksichtigt, bei denen die Strukturberücksichtigung zu anderen Schätzungen als bei deren Nicht-Berücksichtigung führt.

\section{Fragestellung 1}

Es werden zunächst die 98 Mittelwerte und Standardabweichungen berichtet (14 Kategorien $\times 7$ Fächer). Die Interpretation der Mittelwerte orientiert sich an einem Vergleich zwischen Fächern und Kategorien sowie an den Absolutausprägungen der Werte. Der Wert 3 ist hierbei ein Ankerpunkt, da er theoretischer Mittelwert aller Skalen ist. Die Mehrebenenstruktur muss bei der Schätzung der Mittelwerte nicht berücksichtigt werden.

\section{Fragestellung 2}

Um die multidimensionale Datenstruktur (14 Kategorien $\times 7$ Fächer) anschaulich darzustellen, wurde die Korrespondenzanalyse als eine Methode verwendet, anhand derer numerische Informationen in eine grafische Darstellung transformiert werden können. Dabei kann jede Zeile und jede Spalte einer Kreuztabelle als Vektor in einem mehrdimensionalen Raum verstanden werden (Greenacre \& Blasius, 1994). Die sieben untersuchten Domänen stellen beispielsweise die Spalten und die 14 Kategorien die Zeilen einer Kreuztabelle dar, welche die Mittelwerte beinhaltet. So ergibt sich durch die jeweiligen Mittelwerte in den 14 Kategorienspalten für jedes Fach ein spezifisches Profil. Gleichzeitig hat aber auch jede Kategorie, wie zum Beispiel „Stoffmenge“ oder „Veranschaulichung“, ein Profil, welches durch die Werte in den verschiedenen Fächern (7 Spalten) entsteht. Letztlich existiert also für jede untersuchte Domäne ein 14-dimensionales und für jede Kategorie ein 7-dimensionales Profil, welches jeweils als Vektor in einem mehrdimensionalen Raum dargestellt werden kann. Durch die Berechnung der euklidischen Abstände dieser Vektoren bewirkt die Korrespondenzanalyse die Reduktion der Dimensionalität der Profile auf eine zweidimensionale Ebene. Dies ermöglicht eine grafische Darstellung der strukturellen Zusammenhänge im zweidimensionalen Raum (Greenacre \& Blasius, 1994; Kleine, Goetz, Pekrun \& Hall, 2005). Es wurden bivariate Korrespondenzanalysen (symmetrische Normalisierung) mit dem SPSS-Zusatzmodul CORRESPONDENCE durchgeführt, welches von der Data Theory Scaling System Group (DTSS) an der Leiden University (Faculty of Social and Behavioral Sciences) entwickelt wurde. Da in diese Analysen ausschließlich Mittelwerte eingehen, war eine Berücksichtigung der Mehrebenenstruktur nicht notwendig. 


\section{Fragestellung 3}

Um das Ausmaß an Geschlechterunterschieden bezüglich der Angaben zu den Domänencharakteristika einschätzen zu können, wurden für die einzelnen Fächer die Mittelwerte der Beträge der 14 Effektstärken berechnet (mittleres Effektstärkenmaß d). Laut Cohen (1988) kann $d>0.20$ als kleine, $d>0.50$ als mittlere und $d>0.80$ als große Effektstärke betrachtet werden. In die Berechnung von $d$ fließt unter anderem die Standardabweichung ein. Wird bei einer Zwei-Ebenenstruktur die Gesamt-Standardabweichung (über Level 1 und 2) zur Berechnung von $d$ verwendet, so handelt es sich um eine konservative Schätzung, bei der die Effekte in der Regel unterschätzt werden. Bei der vorliegenden Studie wird daher bei jeder berichteten mittleren Effektstärke zusätzlich das ausschließlich auf der Basis der Level 1 Standardabweichung berechnete $d$ angegeben $\left(d_{\text {MS }}\right)$, d. h. bei diesen Effektstärken ist die Mehrebenenstruktur der Daten berücksichtigt.

\section{Ergebnisse}

\section{Gesamtstichprobe}

In Tabelle 2 sind die Mittelwerte und Standardabweichungen für die einzelnen Kategorien jeweils bezogen auf die 7 Unterrichtsfächer für die Gesamtstichprobe dargestellt. Von den insgesamt 98 in der Tabelle dargestellten Mittelwerten werden im Folgenden einige Auffälligkeiten und bemerkenswerte Ausprägungen thematisiert.

Innerhalb der Kategorien zeigen sich relativ große Unterschiede zwischen den Fächern. Die größte Spannweite (= Maximum - Minimum) zeigt sich für die Kategorie „Wichtigkeit des Faches". Musik wird als das mit Abstand unwichtigste Fach eingeschätzt $(M=2.18$; $S D=1.35)$ und Englisch als das mit Abstand wichtigste $(M=4.17 ; S D=1.00)$. Die kleinste Spannweite zeigte sich für die Kategorie „Abwechslung": hier schwankten die Werte lediglich zwischen 2.22 (Mathematik) und 3.11 (Biologie).

Auffällig ist, dass die Mittelwerte bei einigen im Hinblick auf Unterrichtsqualität zentralen Kategorien relativ niedrig ausfallen. So lagen bei der Kategorie „Alltagsbezug“ 4 von 7
Werten unter dem theoretischen Mittelpunkt der Skala von 3.0 - neben Geschichte und Physik insbesondere die Werte für Mathematik $(M=2.20)$ und Musik $(M=2.03)$. Ebenfalls 4 von 7 Werten lagen bei der Kategorie „Meinungsaustausch" unter dem theoretischen Mittelwert $(M=2.52$ für Biologie, $M=2.50$ für Physik, $M=2.31$ für Musik und $M=2.27$ für Mathematik). Bei der Kategorie „Aktuelle Themen" lagen 5 von 7 Werten unter dem theoretischen Mittelpunkt (Biologie: $M=2.75$; Physik: $M=2.43$; Geschichte: $M=2.20$; Musik: $M=1.91$; Mathematik: $M=1.84)$. Auch bei der Kategorie „Veranschaulichung“ lagen 5 von 7 Werten unter dem theoretischen Mittelpunkt - allerdings war hier der geringste Wert von 2.71 noch vergleichsweise hoch. Für die zwei Kategorien „Wichtigkeit Note“ und „Wichtigkeit Fach“ fand sich für alle Fächer ein höherer Wert für die "Wichtigkeit Note“ (jeweils $p<.001$ bei $t$-Test für verbundene Stichproben; auch signifikant bei Berücksichtigung der Mehrebenenstruktur der Daten, siehe oben).

Abbildung 1 zeigt die Ergebnisse der Korrespondenzanalyse für die Gesamtstichprobe. Es wurden die sieben Domänen sowie die 14 Kategorien als Punkte in einer Ebene dargestellt. Ist die Distanz zwischen zwei Punkten gering, so deutet dies auf eine starke dimensionale Übereinstimmung der zugehörigen Konstrukte hin (Kleine et al., 2005).

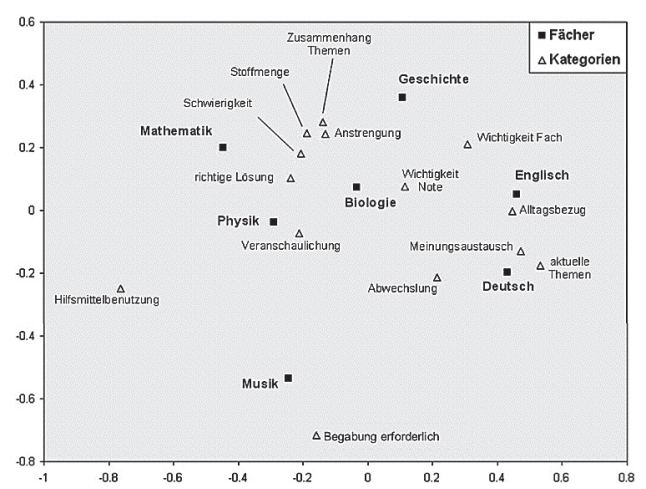

Abbildung 1: Korrespondenzanalyse - Gesamtstichprobe 


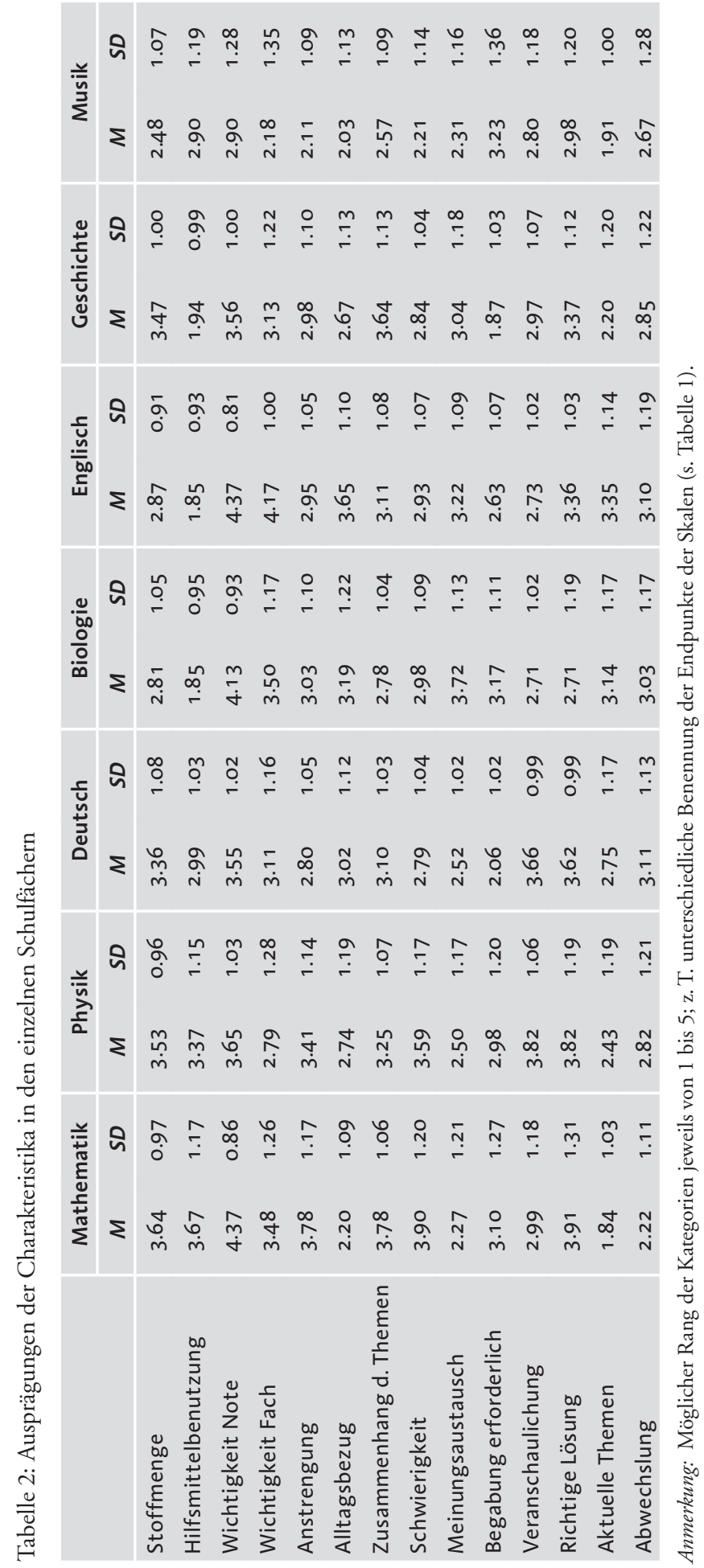


Die Fächer Mathematik und Physik können als ein Cluster betrachtet werden. Ihre Profilpunkte liegen verhältnismäßig nahe beieinander und deutlich weiter links als alle anderen Fächer. Analog können Englisch und Deutsch als ein Cluster angesehen werden. Dabei ist anzumerken, dass sich die zwei erwähnten Cluster im Schaubild gegenüber stehen (horizontale Linie) und einen verhältnismäßig großen Abstand aufweisen, was eine geringe Ähnlichkeit dieser Fächer andeutet. Das Fach Biologie steht zwischen diesen Clustern (Mathematik/Physik, Deutsch/Englisch) und kann weder der einen noch der anderen Seite eindeutig zugeordnet werden. Allerdings weist Biologie einen relativ geringen Abstand zum Fach Physik auf $-d$. h. es gibt Profilähnlichkeiten zwischen beiden Fächern. Die Profilpunkte von Musik und Geschichte liegen sehr weit unten bzw. oben und sind weit entfernt von den Profilpunkten aller anderen Fächer. Somit weisen die Kategorienprofile beider Fächer sehr wenige Ähnlichkeiten zu den Profilen der anderen Fächer auf.

Was die Ähnlichkeiten der Fächer ausmacht bzw. welche Eigenschaften charakterisierend für die Fächer oder Cluster sind, wird in Abbildung 1 deutlich. Je näher eine Kategorie an einem Fach liegt, desto stärker ist diese Kategorie im Gesamtprofil des Faches ausgeprägt. Beispielsweise liegt die Kategorie „Veranschaulichung“" sehr nahe am Fach Physik, was bedeutet, dass „Veranschaulichung“ im Profil des Faches Physik im Vergleich zur Bedeutung von „Veranschaulichung “im Profil anderer Fächer stark ausgeprägt ist. Anders als bei den Mittelwerten zeigt somit die Nähe einer Kategorie zu einem Fach die relative Bedeutung im Profil des Faches an und nicht deren Absolut-Ausprägung. „Veranschaulichung" ist etwa gleich weit von den Fächern Musik, Geschichte, Englisch und Deutsch entfernt, d. h. im Profil dieser Fächer spielt sie eine ähnliche starke Bedeutung, die insgesamt als relativ gering einzuschätzen ist, da „Veranschaulichung "von diesen Fächern jeweils weit entfernt ist.
Um das Cluster Deutsch/Englisch sind vor allem die Kategorien „Abwechslung“, „Meinungsaustausch“, „aktuelle Themen“ und „Alltagsbezug" angeordnet. Somit sind diese kennzeichnend für dieses Cluster. Bei den Fächern Mathematik und Physik gruppieren sich die Kategorien „Veranschaulichung“, „richtige Lösung", „Schwierigkeit", „Stoffmenge“, „Anstrengung" und "Zusammenhang der Themen“. Biologie ist relativ nahe bei diesen Charakteristika und dennoch nicht allzu weit von den Merkmalen des Deutsch/Englisch-Clusters entfernt. Musik zeichnet sich durch hohe Werte in der Kategorie „Begabung erforderlich“ aus, d. h. die Schüler sind der Meinung, dass eher Begabung als Fleiß eine große Rolle für gute Noten im Fach Musik spielt. Die Kategorie „Hilfsmittelbenutzung“ ist sehr weit links angesiedelt und zeigt ähnliche Abstände zu Mathematik, Physik und Musik. Die Kategorie „Wichtigkeit der Note“ liegt relativ zentral zwischen allen Fächern mit Ausnahme des Faches Musik - dieses Fach ist am weitesten entfernt von dieser Kategorie.

\section{Geschlechterspezifische Analysen}

Im Anhang sind für die 7 Fächer jeweils die Mittelwerte und Standardabweichungen der 14 Charakteristika getrennt nach Geschlecht dargestellt. Zudem finden sich dort die Ergebnisse der $t$-Tests zu den Mittelwertsunterschieden ( $t$-Werte und Signifikanzangaben) und die jeweiligen Effektstärken ( $d s)$. Der mit Abstand stärkste Effekt zeigt sich für das Fach Physik $\left(M(|d|)=0.32 ; \mathrm{M}\left(\left|d_{\mathrm{MS}}\right|\right)=0.33\right.$, mittlerer Effekt bei Berücksichtigung der Mehrebenenstruktur), d. h. für das Fach Physik finden sich die insgesamt deutlichsten Unterschiede bei den Angaben von Mädchen und Jungen (v. a. bei Mädchen höhere Werte für "Stoffmenge“, „Anstrengung“ und „Schwierigkeit“; niedrigere Werte bei „Wichtigkeit des Faches“); schwächere und sehr ähnliche mittlere Effekte zeigen sich für Musik $(M(|d|)=0.23$; $M\left(\left|d_{\mathrm{MS}}\right|\right)=0.24$; v. a. bei Mädchen höhere Werte bei „Wichtigkeit des Faches“ und „Wichtig- 
keit der Note") und Deutsch $(M(|d|)=0.22$; $M\left(\left|d_{\text {MS }}\right|\right)=0.23$; v. a. bei Mädchen höhere Werte bei "Wichtigkeit des Faches" und „Wichtigkeit der Note“; niedrigere Werte für „Stoffmenge" und "Schwierigkeit") und relativ schwache Effekte schließlich für Mathematik $\left(M(|d|)=0.16 ; M\left(\left|d_{\mathrm{MS}}\right|\right)=0.16\right)$, Geschichte $\left(M(|d|)=0.12 ; M\left(\left|d_{\mathrm{MS}}\right|\right)=0.13\right)$ und Biologie $\left(M(|d|)=0.12 ; M\left(\left|d_{\mathrm{MS}}\right|\right)=0.13\right)$. Der mit Abstand schwächste Effekt findet sich für das Fach Englisch $\left(M(|d|)=0.06 ; M\left(\left|d_{\mathrm{MS}}\right|\right)=0.06\right)$. Die durchschnittlichen Effekte für die einzelnen Fächer sind insgesamt als klein zu bezeichnen.

Abbildung 2 zeigt die Ergebnisse der Korrespondenzanalyse getrennt für Mädchen und Jungen. Obwohl sich bei den geschlechterspezifischen Korrespondenzanalysen relativ ähnliche Fächercluster für Mädchen und Jungen
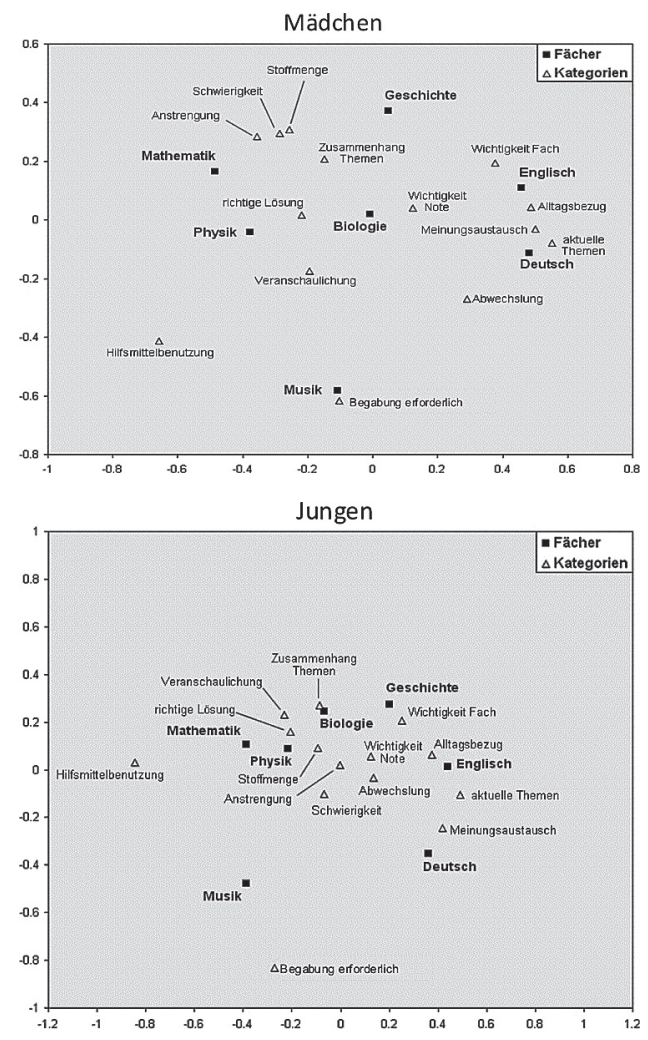

Abbildung 2: Korrespondenzanalyse - geschlechterspezifisch finden, so zeigen sie doch, dass sich die Profile der Cluster z. T. unterscheiden: Die Kategorien „Anstrengung“, „Schwierigkeit“ und „Stoffmenge" liegen bei den Mädchen weiter von Englisch/Deutsch entfernt und dafür näher bei dem Fach Mathematik. Mädchen beschreiben somit die sprachlichen Fächer als weniger anstrengend und schwierig. Zudem nehmen sie die „Stoffmenge“ als geringer wahr.

\section{Diskussion}

Anliegen der Studie ist es, Unterschiede bzw. Gemeinsamkeiten zwischen Fächern im Hinblick auf Charakteristika dieser Fächer aufzudecken. Dabei wird allein von der Perspektive der Schüler ausgegangen. Die Frage, inwieweit es den einzelnen Fächern gelingt, einen Lebens-/Alltagsbezug herzustellen, wie vom "Geist des Lehrplans" gefordert, lässt sich allgemein nicht beantworten. Während dies dem Englisch- wie auch Deutsch-Unterricht gut gelingt, wird eine Kluft zwischen Anspruch und Wirklichkeit gerade im Mathematikunterricht deutlich. Vergleicht man die vorliegenden Ergebnisse nun mit den anfangs skizzierten Bildungszielen, wie in den Lehrplänen formuliert, so ist der gymnasiale Mathematik-Unterricht offenbar weiter von dem Alltagsbezug entfernt, als dies erwünscht ist. Und dies, obwohl die Kategorie „Wichtigkeit" einen relativ hohen Wert erhält. Ähnlich niedrig fällt der Wert für die Kategorie „Meinungsaustausch“ für das Fach Mathematik aus, i. G. zu obiger Formulierung: „Ziel ist es, Mathematik als eine lebendige Wissenschaft möglichst vielen jungen Menschen verständlich zu machen sowie Gespräche darüber anzuregen ....". Auch für die Kategorie „Abwechslung “ fällt hier Mathematik negativ auf.

Dieser Befund bedeutet, für die Lehrerbildung in allen drei Phasen ganz explizit auf die Bedeutung des Faches Mathematik hinzuweisen. Pädagogen sprechen hier vom „Sitz im Leben“, der Schülern beim Lernen klar werden muss. Eine konkrete Umsetzungsmaßnahme könnte sein, wenn sich Lehrkräfte in ihrem Unterricht an Ansätzen des situierten Lernens (vgl. 
Schnotz, 2006) orientieren. Eine zentrale Annahme ist, dass erfolgreiches Lernen kontextgebunden ist, d. h. eingebunden in konkreten Situationen und Kontexten. Wenn hierauf bei der Aufgabenstellung Mathematiklehrer achten, dass sie solche Aufgaben auswählen, die der Lebenswelt ihrer Schüler entstammen, dann ist ihr Fach näher am „Leben“ verortet.

Dieser Befund mag genügen, um die Forderung des Expertenansatzes zu unterstreichen, genauer Fachkulturen unter die Lupe zu nehmen, wenn es um die Bedeutung pädagogischen Wissens für die Expertise eines Lehrers geht.

Die zweite Frage nach der Fachkultur zwischen verschiedenen Fachkombinationen zeigt deutliche Unterschiede. Die Fächer Mathematik und Physik können als ein Cluster betrachtet werden. Ihre Profilpunkte liegen verhältnismäßig nahe beieinander. Dabei gruppieren sich die Kategorien „Veranschaulichung“, „richtige Lösung“, „Schwierigkeit“, „Stoffmenge“, „Anstrengung" und „Zusammenhang der Themen“. Wie schon bei Merzyn (2008) festgestellt, gelten Mathematik und Physik als schwierige Fächer. Dadurch, dass auch Geschichte mit in vorliegende Befragung miteinbezogen wurde, wird auch hier ein so erstmal nicht erwartetet hoher Wert sichtbar. Analog können Englisch und Deutsch als ein Cluster angesehen werden. Kennzeichnend für dieses Cluster sind „Abwechslung“, „Meinungsaustausch“, „aktuelle Themen“, und „Alltagsbezug“ angeordnet.

Die immer wieder berichteten gravierenden geschlechtsspezifischen Unterschiede zwischen den Fächern zeigen sich hier nur bedingt. Zwar kann Physik als ein Jungenfach und Deutsch als Mädchenfach bestätigt werden, doch in Englisch unterscheiden sich Jungen und Mädchen kaum. Auf diese Stellung des Faches Englisch weist schon die oben zitierte neuere Studie von Roisch (2003). Beim Fach Mathematik wird deutlich, dass hier das Bild einer einstmals vorherrschenden Jungendomäne so nicht mehr haltbar ist. Insgesamt zeigt die Studie klar, dass in weiteren Analysen über Unterrichtsfächer diese möglichst gemeinsam miteinbezogen werden sollten.

\section{Literatur}

Alt-Stutterheim, W. v. (1980). Die Kollegstufe im Urteil von Kollegiaten. München: Ehrenwirth.

Bachmair, G. (1969). Einstellungen von Schülern zum Lehrer und zum Unterrichtsfach. Inaugural-Dissertation der Philosophischen Fakultät der Friedrich-AlexanderUniversität Erlangen-Nürnberg.

Baumert, J., Bos, W. \& Watermann, R. (1999). TIMSS/III. Schülerleistungen in Mathematik und den Naturwissenschaften am Ende der Sekundarstufe II im internationalen Vergleich. Berlin: Max-Planck-Institut für Bildungsforschung.

Baumert, J., Lehmann, R. u. a. (1997). TIMSS - Mathematisch-naturwissenschaftlicher Unterricht im internationalen Vergleich. Opladen: Leske + Budrich.

Bayerisches Staatsministerium für Unterricht und Kultus (2003). Lehrplan für das Gymnasium in Bayern. Wolnzach: Kastner AG.

Brickhouse, N. W. (1990). Teachers' beliefs about the nature of science and their relationship to classroom practice. Journal of Teacher Education, 41, 53-62.

Bromme, R. (1992). Der Lehrer als Experte. Zur Psychologie des professionellen Wissens. Bern: Huber.

Bromme, R \& Haag, L. (2004). Forschung zur Lehrerpersönlichkeit. In W. Helsper \& J. Böhme (Hrsg.), Handbuch der Schulforschung (S. $777-793)$. Wiesbaden: Verlag für Sozialwissenschaften.

Cohen, J. (1988). Statistical power analysis for the behavioural sciences (2nd ed.). Hillsdale, NJ: Erlbaum.

Demuth, R. (2006). Chemie. In K.-H. Arnold, U. Sandfuchs \& J. Wiechmann (Hrsg.), Handbuch Unterricht (S. 476 -480). Bad Heilbrunn: Klinkhardt.

Deutsches PISA-Konsortium (2001). PISA 2000. Basiskompetenzen von Schülerinnen und Schülern im internationalen Vergleich. Leske + Budrich: Opladen.

Euler, M. (1982). Physikunterricht-Anspruch und Realität. Frankfurt: Lang.

Gellert, U. (2006). Mathematik. In K.-H. Arnold, U. Sandfuchs \& J. Wiechmann (Hrsg.), Handbuch Unterricht (S. 467 -471). Bad Heilbrunn: Klinkhardt.

Götz, T., Lohrmann, K., Ganser, B. \& Haag, L. (2005): Einsatz von Unterrichtsmethoden - Konstanz oder Wandel? Empirische Pädagogik, 19, 342-360.

Greenacre, M. \& Blasius, J. (1994). Correspondence analysis in the social sciences. San Diego: Academic Press.

Hoffmann, L. \& Lehrke, M. (1985). Eine Zusammenstellung erster Ergebnisse aus der Querschnitterhebung 1984 über Schüerinteressen an Physik und Technik vom 5. bis 10. Schuljahr. Kiel: IPN.

Jäger-Flor, D. \& Jäger, R. S. (2008). Bildungsbarometer zum Thema "Mathematik". Ergebnisse, Bewertungen und Perspektiven. Landau: Verlag Empirische Pädagogik.

Kleine, M., Goetz, T., Pekrun, R. \& Hall, N. (2005). The structure of students' emotions experienced during an academic achievement test. International Reviews on Mathematical Education, 37(3), 221 - 225.

Köller, O., Baumert, J. \& Neubrand, J. (2000). Epistemologische Überzeugungen und Fachverständnis im Mathematik- und Physikunterricht. In J. Baumert, W. Bos, \& R. Lehmann (Hrsg), Dritte Internationale Mathematik- und Naturwissenschaftsstudie - Mathematische und naturwissenschaftliche Bildung am Ende der Schullaufbahn. Opladen: Leske + Budrich.

Krause, F. \& Reiners-Logothetidou, A. (1979). Der bundesweite Studieneingangstest Physik 1978. Physikalische Blätter, 35, 495-510. 
Labudde, P. (2006). Fachunterricht und fächerübergreifender Unterricht: Grundlagen. In K.-H. Arnold, U. Sandfuchs \& J. Wiechmann (Hrsg.), Handbuch Unterricht (S. 441-447). Bad Heilbrunn: Klinkhardt.

Labudde, P. \& Pfluger, D. (1999). Physikunterricht in der Sekundarstufe II. Zeitschrift Didaktik Naturwissenschaften 5(2), 33-50.

Mayring, P. \& Gläser-Zikuda, M. (2005): Die Praxis der qualitativen Inhaltsanalyse. Weinheim (Beltz/UTB).

Merzyn, G. (2008). Naturwissenschaften, Mathematik, Technik - immer unbeliebter? Baltmannsweiler: Schneider Verlag Hohengehren.

Muckenfuß, H. (1995). Lernen im sinnstiftenden Kontext. Berlin: Cornelsen.

Muthén, L. K. \& Muthén, B. O. (1998-2008). Mplus user's guide. Fifth edition. Los Angeles, CA: Muthén \& Muthén.

Reinhold, P. (2006). Physik. In K.-H. Arnold, U. Sandfuchs \& J. Wiechmann (Hrsg.), Handbuch Unterricht (S. 480-484). Bad Heilbrunn: Klinkhardt.

Roisch, H. (2003). Geschlechtsspezifische Interessengebiete und Interessenpräferenzen. In M. Stürzer, H. Roisch, A. Hunze \& W. Cornelißen (Hrsg.), Geschlechterverhältnisse in der Schule (S.123-150). Opladen: Leske + Budrich.

Schnell, J. (2009). CHADO-Studie. Charakterisierung von Schulfächern. Eine qualitative Interviewstudie. Wissenschaftliche Qualifikationsarbeit für die Zulassung zum Staatsexamen (Gymnasiales Lehramt). Universität Konstanz.

Schnotz, W. (2006). Pädagogische Psychologie. Weinheim: Beltz.

Seidel, T. \& Prenzel M. (2004). Muster unterrichtlicher Aktivitäten im Physikunterricht. In J. Doll \& M. Prenzel (Hrsg.), Bildungsqualität von Schule (S. 177-194). Münster: Waxmann.

Shell-Jugendwerk (1992). Jugend '92. Opladen: Leske + Budrich.

Shulman, L. S. (1986). Those who understand. Knowledge growth in teaching. Educational Researcher 15, 4-14.

Slavin, R. E. (1995). Cooperative learning: Theory, research, and practice. (2nd ed.). Englewood Cliffs, NJ: PrenticeHall.

Stanat, P. \& Kunter, M. (2001). Geschlechterunterschiede in Basiskompetenzen. In Deutsches PISA-Konsortium (Hrsg.), PISA 2000 (S. 251 -269). Opladen: Leske + Budrich.
Stern, L.W. (1905). Über Beliebtheit und Unbeliebtheit der Schulfächer. Zeitschrift für pädagogische Psychologie und Jugendkunde, 7, 267-296.

Stevens, R. Wineburg, S., Herrenkohl, R. L. \& Bell, P. (2005). Comparative understanding of school subjects: past, present and future. Review of Educational Research, 75 (2), 125-157.

Tenorth, H.-E. (1999). Unterrichtsfächer - Möglichkeit, Rahmen und Grenze. In I. F. Goodson, S. Hopmann \& K. Riquarts (Hrsg.), Das Schulfach als Handlungsrahmen. Vergleichende Untersuchung zur Geschichte und Funktion der Schulfächer (S. 191 - 207). Köln/Weimar/ Wien: Böhlau Verlag.

Todt, E. (1978). Das Interesse. Bern: Huber.

Zimmer, K., Burba, D. \& Rost, J. (2004). Kompetenzen von Jungen und Mädchen. In PISA-Konsortium Deutschland (Hrsg.), PISA 2003 (S. 211 - 223). Münster: Waxmann.

\section{Prof. Dr. Ludwig Haag}

Universität Bayreuth

Kulturwissenschaftliche Fakultät

Lehrstuhl für Schulpädagogik

Universitätsstraße 30

D-95440 Bayreuth

Tel.: ++49(o)9 21 55-41 05

Fax: ++49(o)9 21 55-42 22

E-Mail: ludwig.haag@uni-bayreuth.de

Prof. Dr. Thomas Götz

Universität Konstanzl

Pädagogische Hochschule Thurgau

Geisteswissenschaftliche Sektion

Erziehungswissenschaft/

Empirische Bildungsforschung

Universitätsstraße 10

D-78457 Konstanz

Tel.: ++49(0)75 31-88-41 44

Fax: ++49(o)75 31-88-4350

E-Mail: thomas.goetz@uni-konstanz.de 
Mathe ist schwierig und Deutsch aktuell 45

\section{Anhang}

\section{Ausprägungen der Schulfach-Charakteristika differenziert nach Geschlecht}

\begin{tabular}{|c|c|c|c|c|c|c|}
\hline \multirow{2}{*}{ Kategorie } & \multicolumn{2}{|c|}{ weiblich } & \multicolumn{2}{|c|}{ männlich } & \multirow[b]{2}{*}{$t$} & \multirow[b]{2}{*}{$d$} \\
\hline & $M$ & $S D$ & $M$ & $S D$ & & \\
\hline \multicolumn{7}{|l|}{ Mathematik } \\
\hline Stoffmenge & 3.72 & 0.88 & 3.53 & 1.05 & $3 \cdot 39^{* *}$ & 0.20 \\
\hline Hilfsmittelbenutzung & 3.64 & 1.16 & 3.71 & 1.18 & -1.10 & -0.06 \\
\hline Wichtigkeit Note & $4 \cdot 37$ & 0.84 & 4.38 & 0.86 & -0.32 & -0.02 \\
\hline Wichtigkeit Fach & $3 \cdot 31$ & 1.26 & 3.68 & 1.23 & $-5.07^{* * *}$ & -0.29 \\
\hline Anstrengung & 3.95 & 1.07 & 3.59 & 1.25 & $5 \cdot 3^{8 * * *}$ & 0.31 \\
\hline Alltagsbezug & 2.12 & 1.02 & 2.31 & 1.17 & $-2.92^{* *}$ & -0.17 \\
\hline Zusammenhang der Themen & 3.81 & 1.04 & 3.74 & 1.08 & 1.19 & 0.07 \\
\hline Schwierigkeit & 4.12 & 1.07 & 3.64 & 1.29 & $6.99^{* * *}$ & 0.41 \\
\hline Meinungsaustausch & 2.22 & 1.24 & 2.33 & 1.18 & -1.58 & -0.09 \\
\hline Begabung erforderlich & 3.15 & 1.26 & 3.03 & 1.28 & 1.61 & 0.09 \\
\hline Veranschaulichung & 2.97 & 1.18 & 3.01 & 1.19 & -0.72 & -0.04 \\
\hline Richtige Lösung & 3.86 & 1.33 & 3.97 & 1.29 & -1.46 & -0.08 \\
\hline Aktuelle Themen & 1.76 & 0.95 & 1.94 & 1.11 & $-3.04^{* *}$ & -0.18 \\
\hline Abwechslung & 2.14 & 1.07 & 2.33 & 1.15 & $-2.90^{* *}$ & -0.17 \\
\hline \multicolumn{7}{|l|}{ Physik } \\
\hline Stoffmenge & 3.72 & 0.87 & 3.28 & 1.01 & $8.08 * * *$ & 0.47 \\
\hline Hilfsmittelbenutzung & $3 \cdot 30$ & 1.15 & 3.44 & 1.15 & $-2.08^{*}$ & -0.12 \\
\hline Wichtigkeit Note & 3.56 & 1.01 & 3.77 & 1.04 & $-3 \cdot 50 * * *$ & -0.20 \\
\hline Wichtigkeit Fach & 2.41 & 1.15 & 3.24 & 1.29 & $-11.88 * * *$ & -0.69 \\
\hline Anstrengung & 3.67 & 1.06 & 3.09 & 1.14 & $9.19^{* * *}$ & 0.53 \\
\hline Alltagsbezug & 2.53 & 1.14 & 2.99 & 1.20 & $-6.82^{* *}$ & -0.39 \\
\hline Zusammenhang der Themen & 3.16 & 1.05 & 3.36 & 1.07 & $-3.29 * *$ & -0.19 \\
\hline Schwierigkeit & 3.93 & 1.02 & 3.19 & 1.21 & $11.40^{* * *}$ & 0.67 \\
\hline Meinungsaustausch & 2.37 & 1.15 & 2.67 & 1.17 & $-4.55^{* * *}$ & -0.26 \\
\hline Begabung erforderlich & 3.09 & 1.20 & 2.85 & 1.18 & $3.61 * * *$ & 0.21 \\
\hline Veranschaulichung & 3.80 & 1.07 & 3.85 & 1.04 & -0.91 & -0.05 \\
\hline Richtige Lösung & 3.69 & 1.24 & 3.98 & 1.11 & $-4.26 * * *$ & -0.24 \\
\hline Aktuelle Themen & 2.34 & 1.15 & 2.54 & 1.23 & $-2.85^{* *}$ & -0.16 \\
\hline Abwechslung & 2.68 & 1.21 & 2.98 & 1.19 & $-4 \cdot 31 * * *$ & -0.25 \\
\hline \multicolumn{7}{|l|}{ Biologie } \\
\hline Stoffmenge & 3.45 & 1.03 & 3.23 & 1.13 & $3.48 * *$ & 0.20 \\
\hline Hilfsmittelbenutzung & 2.95 & 1.02 & 3.03 & 1.04 & -1.24 & -0.07 \\
\hline Wichtigkeit Note & 3.64 & 0.97 & 3.44 & 1.07 & $3 \cdot 39^{* *}$ & 0.20 \\
\hline Wichtigkeit Fach & 3.14 & 1.16 & 3.09 & 1.16 & 0.75 & 0.04 \\
\hline Anstrengung & 2.85 & 1.03 & 2.74 & 1.06 & 1.78 & 0.10 \\
\hline Alltagsbezug & 3.07 & 1.13 & 2.98 & 1.10 & 1.30 & 0.08 \\
\hline Zusammenhang der Themen & 3.17 & 1.00 & 3.01 & 1.05 & $2.77^{* *}$ & 0.16 \\
\hline Schwierigkeit & 2.91 & 1.04 & 2.64 & 1.01 & $4 \cdot 31 * * *$ & 0.25 \\
\hline Meinungsaustausch & 2.47 & 1.02 & 2.58 & 1.02 & -1.71 & -0.10 \\
\hline Begabung erforderlich & 2.04 & 0.98 & 2.07 & 1.05 & -0.57 & -0.03 \\
\hline Veranschaulichung & 3.72 & 0.97 & 3.60 & 1.01 & $2.04^{*}$ & 0.12 \\
\hline Richtige Lösung & 3.60 & 0.95 & 3.64 & 1.05 & -0.56 & -0.03 \\
\hline Aktuelle Themen & 2.82 & 1.19 & 2.67 & 1.15 & 2.23 & 0.13 \\
\hline Abwechslung & 3.16 & 1.12 & 3.05 & 1.15 & 1.68 & 0.10 \\
\hline \multicolumn{7}{|l|}{ Deutsch } \\
\hline Stoffmenge & 2.62 & 0.97 & 3.04 & 1.08 & $-7.22^{* * *}$ & -0.41 \\
\hline Hilfsmittelbenutzung & 1.88 & 0.96 & 1.82 & 0.94 & 1.19 & 0.07 \\
\hline Wichtigkeit Note & $4 \cdot 30$ & 0.83 & 3.94 & 1.00 & $6.64^{* * *}$ & 0.39 \\
\hline Wichtigkeit Fach & 3.69 & 1.08 & 3.28 & 1.24 & $6.18 * * *$ & 0.36 \\
\hline Anstrengung & 2.88 & 1.08 & 3.21 & 1.10 & $-5.27^{\star * *}$ & -0.30 \\
\hline
\end{tabular}




\begin{tabular}{|c|c|c|c|c|c|c|}
\hline \multirow[b]{2}{*}{ Kategorie } & \multicolumn{2}{|c|}{ weiblich } & \multicolumn{2}{|c|}{ männlich } & \multirow[b]{2}{*}{$t$} & \multirow[b]{2}{*}{$d$} \\
\hline & $M$ & $S D$ & $M$ & $S D$ & & \\
\hline Alltagsbezug & 3.34 & 1.16 & 3.01 & 1.27 & $4.78^{* * *}$ & 0.27 \\
\hline Zusammenhang der Themen & 2.83 & 1.02 & 2.72 & 1.06 & 1.80 & 0.10 \\
\hline Schwierigkeit & 2.77 & 1.07 & 3.23 & 1.07 & $-7.49^{* * *}$ & -0.43 \\
\hline Meinungsaustausch & 3.82 & 1.09 & 3.62 & 1.16 & $3.12^{* \star}$ & 0.18 \\
\hline Begabung erforderlich & 3.18 & 1.09 & 3.15 & 1.14 & 0.32 & 0.02 \\
\hline Veranschaulichung & 2.74 & 1.00 & 2.69 & 1.05 & 0.87 & 0.05 \\
\hline Richtige Lösung & 2.73 & 1.16 & 2.69 & 1.22 & 0.65 & 0.04 \\
\hline Aktuelle Themen & 3.21 & 1.12 & 3.04 & 1.23 & $2.49^{\star}$ & 0.14 \\
\hline Abwechslung & 3.19 & 1.12 & 2.82 & 1.20 & $5 \cdot 53^{* * *}$ & 0.32 \\
\hline \multicolumn{7}{|l|}{ Englisch } \\
\hline Stoffmenge & 2.82 & 0.85 & 2.93 & 0.97 & $-2.00^{*}$ & -0.12 \\
\hline Hilfsmittelbenutzung & 1.87 & 0.96 & 1.83 & 0.90 & .071 & 0.04 \\
\hline Wichtigkeit Note & 4.43 & 0.78 & 4.31 & 0.82 & $2.63^{* *}$ & 0.15 \\
\hline Wichtigkeit Fach & 4.20 & 0.98 & 4.12 & 1.02 & 1.43 & 0.08 \\
\hline Anstrengung & 2.94 & 1.05 & 2.96 & 1.04 & -0.25 & -0.01 \\
\hline Alltagsbezug & 3.71 & 1.09 & 3.57 & 1.11 & $2.31^{*}$ & 0.13 \\
\hline Zusammenhang der Themen & 3.11 & 1.05 & 3.11 & 1.12 & 0.03 & 0.00 \\
\hline Schwierigkeit & 2.88 & 1.06 & 2.98 & 1.08 & -1.68 & -0.10 \\
\hline Meinungsaustausch & 3.22 & 1.09 & 3.23 & 1.09 & -0.08 & 0.00 \\
\hline Begabung erforderlich & 2.63 & 1.06 & 2.61 & 1.09 & 0.30 & 0.02 \\
\hline Veranschaulichung & 2.72 & 1.03 & 2.74 & 1.01 & -0.36 & -0.02 \\
\hline Richtige Lösung & 3.33 & 1.05 & 3.41 & 1.02 & -1.35 & -0.08 \\
\hline Aktuelle Themen & 3.35 & 1.14 & 3.34 & 1.14 & 0.21 & 0.01 \\
\hline Abwechslung & 3.14 & 1.21 & 3.06 & 1.17 & 1.27 & 0.07 \\
\hline \multicolumn{7}{|l|}{ Geschichte } \\
\hline Stoffmenge & 3.55 & 0.97 & 3.38 & 1.04 & $2.92^{* *}$ & 0.17 \\
\hline Hilfsmittelbenutzung & 1.91 & 0.96 & 1.97 & 1.03 & -1.19 & -0.07 \\
\hline Wichtigkeit Note & 3.57 & 0.98 & 3.55 & 1.02 & 0.26 & 0.02 \\
\hline Wichtigkeit Fach & 3.08 & 1.21 & 3.21 & 1.23 & -1.85 & -0.11 \\
\hline Anstrengung & 3.04 & 1.10 & 2.92 & 1.08 & 1.89 & 0.11 \\
\hline Alltagsbezug & 2.64 & 1.11 & 2.71 & 1.16 & -1.15 & -0.07 \\
\hline Zusammenhang der Themen & 3.54 & 1.14 & 3.77 & 1.11 & $-3 \cdot 54^{* * *}$ & -0.20 \\
\hline Schwierigkeit & 2.90 & 1.06 & 2.77 & 1.01 & $2.03^{*}$ & 0.12 \\
\hline Meinungsaustausch & 2.92 & 1.18 & 3.19 & 1.16 & $-4.02 * * *$ & -0.23 \\
\hline Begabung erforderlich & 1.86 & 1.04 & 1.89 & 1.03 & -.045 & -0.03 \\
\hline Veranschaulichung & 2.88 & 1.09 & 3.08 & 1.04 & $-3 \cdot 30^{* *}$ & -0.19 \\
\hline Richtige Lösung & 3.36 & 1.11 & 3.39 & 1.12 & -0.46 & -0.03 \\
\hline Aktuelle Themen & 2.16 & 1.16 & 2.25 & 1.24 & -1.22 & -0.07 \\
\hline Abwechslung & 2.71 & 1.25 & 3.01 & 1.16 & $-4.40^{* * *}$ & -0.25 \\
\hline \multicolumn{7}{|l|}{ Musik } \\
\hline Stoffmenge & 2.41 & 0.95 & 2.56 & 1.19 & $-2.46^{\star}$ & -0.15 \\
\hline Hilfsmittelbenutzung & 2.99 & 1.19 & 2.79 & 1.17 & $2.88^{\star *}$ & 0.17 \\
\hline Wichtigkeit Note & 3.24 & 1.15 & 2.50 & 1.32 & $10.33^{* * *}$ & 0.60 \\
\hline Wichtigkeit Fach & 2.40 & 1.38 & 1.91 & 1.27 & $6.44^{* * *}$ & 0.37 \\
\hline Anstrengung & 2.10 & 1.02 & 2.11 & 1.17 & -0.23 & -0.01 \\
\hline Alltagsbezug & 2.15 & 1.13 & 1.87 & 1.09 & $4 \cdot 36^{* * *}$ & 0.25 \\
\hline Zusammenhang der Themen & 2.70 & 1.03 & 2.42 & 1.15 & $4.47^{\star \star *}$ & 0.26 \\
\hline Schwierigkeit & 2.10 & 1.02 & 2.35 & 1.25 & $-3.71^{* * *}$ & -0.22 \\
\hline Meinungsaustausch & 2.34 & 1.15 & 2.27 & 1.18 & 1.05 & 0.06 \\
\hline Begabung erforderlich & 3.19 & 1.33 & 3.28 & 1.41 & -1.12 & -0.06 \\
\hline Veranschaulichung & 2.95 & 1.17 & 2.62 & 1.16 & $4.85^{* * *}$ & 0.28 \\
\hline Richtige Lösung & 3.13 & 1.16 & 2.80 & 1.22 & $4.91^{* * *}$ & 0.28 \\
\hline Aktuelle Themen & 1.97 & 0.98 & 1.84 & 1.01 & $2.24^{*}$ & 0.13 \\
\hline Abwechslung & 2.85 & 1.27 & 2.45 & 1.27 & $5.47^{* * *}$ & 0.31 \\
\hline
\end{tabular}

Anmerkung: $t$-Werte: ${ }^{*} p<.05 ;{ }^{* *} p<.01 ;{ }^{* * *} p<.001$ 